\title{
Editorial
}

\section{Photomedicine and Photo Nanosystems}

\author{
Rodica-Mariana Ion, ${ }^{1}$ Tebello Nyokong, ${ }^{2}$ G. Gyulkhandanyan, ${ }^{3}$ and Danuta Wrobel ${ }^{4}$ \\ ${ }^{1}$ Analytical Department, National Institute for Chemical and Petrochemical Research, 060021 Bucharest, Romania \\ ${ }^{2}$ Chemistry Department, Rhodes University, Grahamstown 6140, South Africa \\ ${ }^{3}$ Institute of Biochemistry, National Academy of Sciences of Armenia, 0014 Yerevan, Armenia \\ ${ }^{4}$ Department of Molecular Physics, Institute of Physics, Poznan University of Technology, 60-965 Poznań, Poland
}

Correspondence should be addressed to Rodica-Mariana Ion, rodica_ion2000@yahoo.co.uk

Received 15 March 2012; Accepted 15 March 2012

Copyright (C) 2012 Rodica-Mariana Ion et al. This is an open access article distributed under the Creative Commons Attribution License, which permits unrestricted use, distribution, and reproduction in any medium, provided the original work is properly cited.

Considered as a key technology of the 21st century, the nanotechnology is considered a useful tool that may help to develop new and efficient applications, especially in nanomedicine.

Nanomedicine exploits the physical, chemical, and biological properties of materials at the nanometer scale. It is expected to have its substantial impact on preventive medicine, diagnostics, medical imaging, therapy, and drug delivery.

In this special issue of International Journal of Photoenergy, there were presented some basic and engineering researches in the field of nanomedicine and the related biotherapeutic discovery.

This issue covered current topics and recent progress in targeted nanomedicine and therapeutic concepts, photodynamic therapy and regenerative medicine.

The Authors have submitted original research and articles describing the latest scientific and technological research results in the following topics. The topics included the following:

(i) nanostructures and biomaterials,

(ii) nanomedicine,

(iii) nanobiotechnology and nanomedicine,

(iv) drug discovery and development.

In the name of the editorial team, I want to express my appreciation about the quality, originality, and novelty of works submitted by the authors, and in this respect, we welcome the proposal of the journal to publish a special number dedicated to the field of nanomedicine.
Rodica-Mariana Ion Tebello Nyokong G. Gyulkhandanyan Danuta Wrobel 


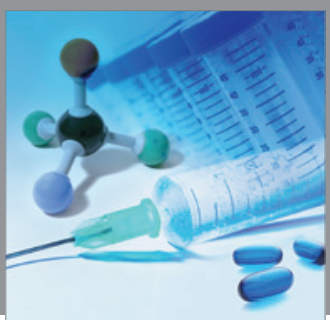

International Journal of

Medicinal Chemistry

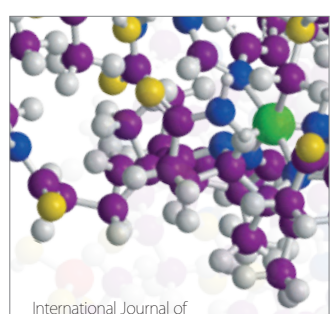

Carbohydrate Chemistry

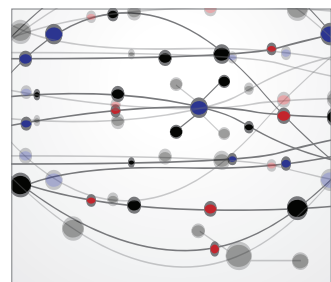

The Scientific World Journal
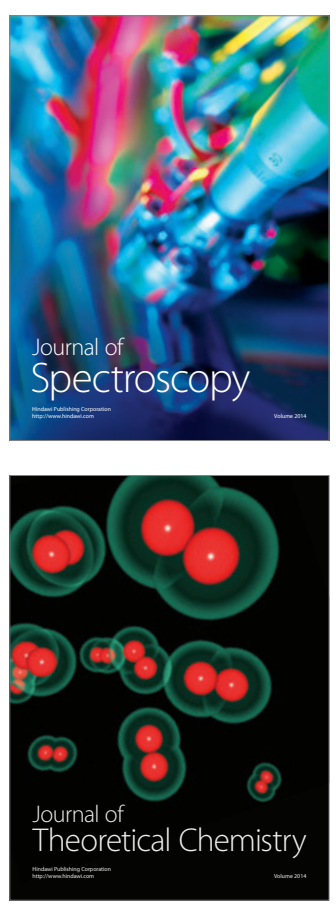
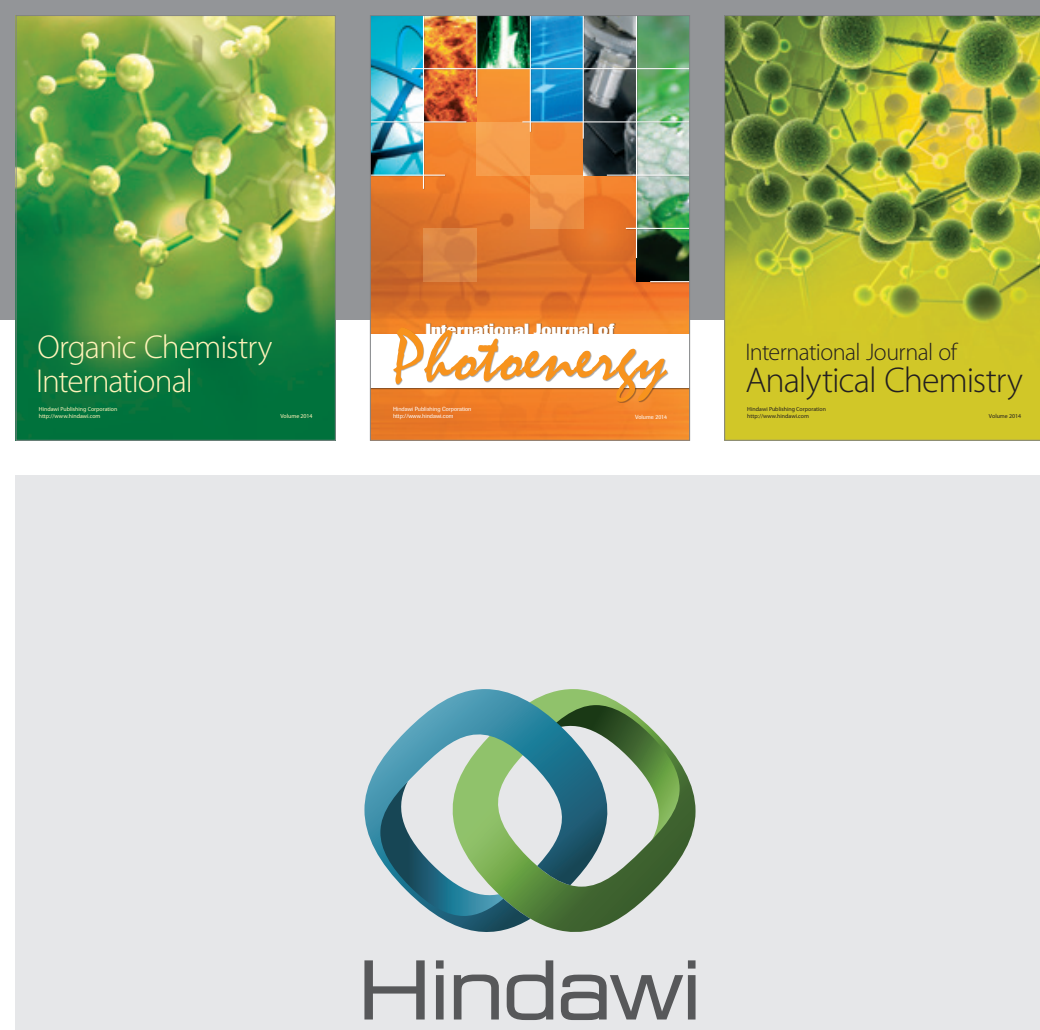

Submit your manuscripts at

http://www.hindawi.com
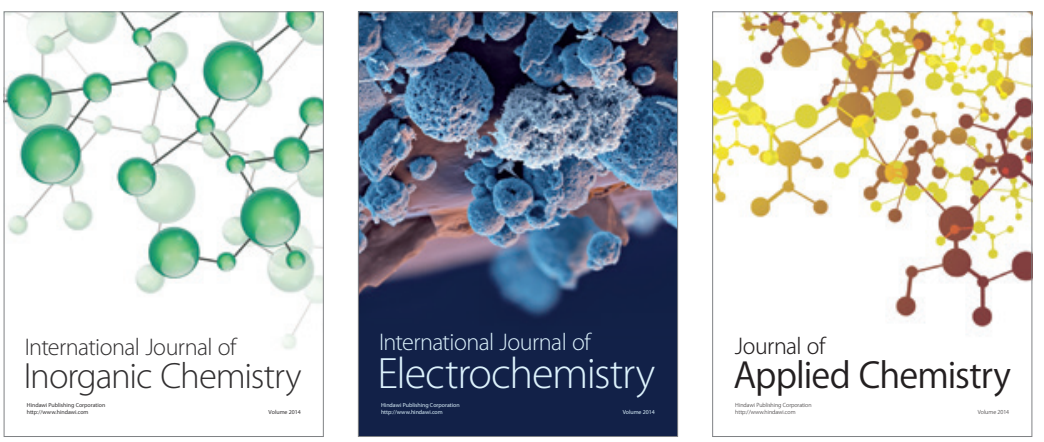

Journal of

Applied Chemistry
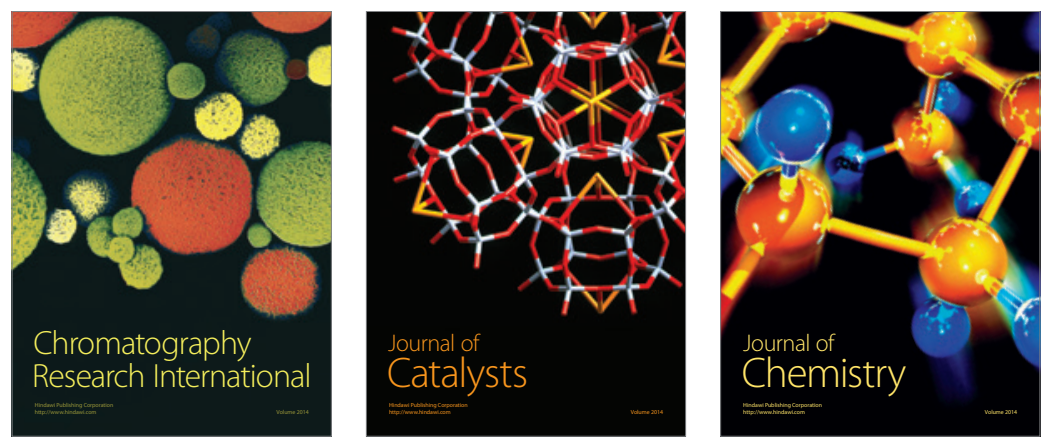
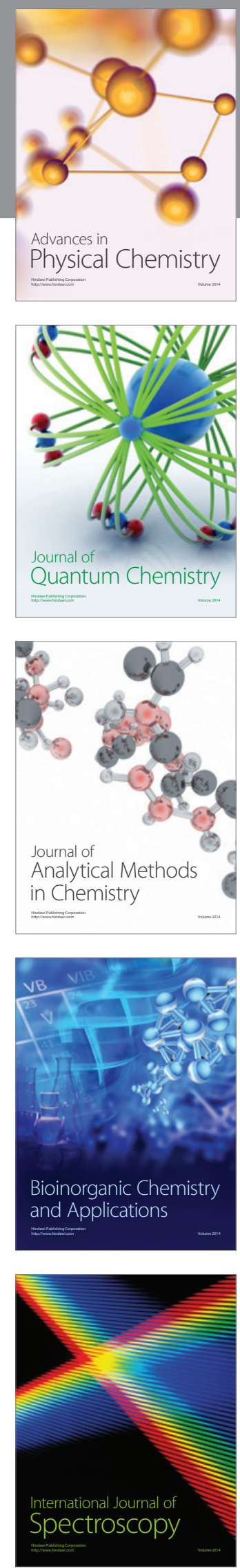